\title{
Prevalênçia de Toxoplasma gondii em gestantes atendidas pelo Sistema Único de Saúde
}

\author{
Toxoplasma gondii prevalence in pregnant women attended by the Unified Health System
}

\author{
Thaís Laila Nascimentoa, Camila Mariangela Pacheco ${ }^{b}$, Fabrízio Furtado de Sousac \\ a Graduada em Ciências Biológicas pela Universidade do Estado de Minas Gerais, Unidade Divinópolis. \\ b Bióloga. Mestre em Biologia Animal. Docente do Curso de Ciências Biológicas da Universidade do Estado de Minas Gerais, Unidade Divinópolis. \\ Agrônomo. Mestre em Genética e Melhoramento. Docente dos Cursos de Ciências Biológicas e Educação Física da Universidade do Estado de Minas Gerais, \\ Unidade Divinópolis.
}

RESUMO

Introdução: A toxoplasmose é uma das zoonoses mais disseminadas do mundo, conhecida popularmente como a "doença do gato". A infecção materna primária adquirida durante a gestação, é de elevada importância podendo resultar em infecção com graves sequelas para o feto.

Objetivo: Analisar a prevalência do Toxoplasma gondii em gestantes atendidas Sistema Único de Saúde (SUS), na cidade de Divinópolis, Minas Gerais, Brasil, durante os anos de 2013 e 2014.

Materiais e Métodos: Foi feito um estudo transversal retrospectivo a partir da analise da prevalência do Toxoplasma gondii. Os dados foram estatisticamente analisados segundo o teste $\chi^{2}$.

Resultados: As prevalências observadas foram de 45 e 38\% em 2013 e 2014, respectivamente, sendo encontrada diferença significativa entre os dois anos $(p<0,05)$. Na análise da distribuição dos registros obtidos por regiões no município, foi observada maior frequência nas regiões Sudoeste distante, Noroeste rural, Noroeste distante e Oeste. Não houve diferença significativa entre as regiões atingindo prevalência de até $58,6 \%$. A soropositividade também foi relacionada com as categorias de idade, sendo mais frequente entre as gestantes de15 a $\geq 35$ anos. Em 2013 houve relação entre a idade e a soroprevalência $(p<0,05)$.

Conclusão: Verificou-se a prevalência de soropositividade para Toxoplasma gondii em 45\% das gestantes, em 2013, em 38\%, em 2014. Estas prevalências, encontradas na cidade de Divinópolis-MG, são semelhante ás observadas em outros estudos no Brasil, ressaltando a importância de medidas de prevenção, que devem ser intensificadas durante o acompanhamento gestacional, para diminuir a soroconversão e a transmissão fetal.

Palavras-chave: Toxoplasma gondii; toxoplasmose; gestantes.

\section{ABSTRACT}

Introduction: Toxoplasmosis is one of the most widespread zoonosis in the world, known popularly as the "cat disease". Primary
maternal infection acquired during pregnancy is of great importance and may result in infection with serious consequences for the fetus.

Objective: To assess the prevalence of Toxoplasma gondii infection in pregnant women attended by the Unified Health System (SUS), in Divinópolis, Minas Gerais, Brazil, during the years 2013 and 2014.

Materials and Methods: A retrospective cross-sectional study was performed from the analysis of the prevalence of Toxoplasma gondii. Data were statistically analyzed using the Chi square test.

Results: The prevalence were 45 and 38\% in 2013 and 2014, respectively, and significant difference between the two years $(p<0.05)$ were found. When analyzing the distribution of the records obtained by regions of the city, we have observed the highest frequencies in regions far west, rural Northwest, far Northwest and West. There was no significant difference between regions reaching a prevalence of up to $58.6 \%$. The seropositivity was also related to the categories of age, being more frequent among pregnant women of 15 to $\geq 35$ years. In 2013 there was also a relationship between age and the seropositivity $(p<0.05)$.

Conclusion: The prevalence of seropositivity for Toxoplasma gondii was of 45\% in pregnant women in 2013 and $38 \%$ in 2014 . These prevalences found in Divinópolis-MG are similar to those observed in other studies in Brazil, emphasizing the importance of preventive measures, which should be intensified during pregnancy to reduce the seroconversion and fetal transmission.

Keywords: Toxoplasma gondii; toxoplasmosis; pregnant women

\section{Correspondência:}

THAÍS LAILA NASCIMENTO

Av. Paraná, 3001 - Jardim Belvedere

35501-170 Divinópolis, MG, Brasil

E-mail: thaislaina@gmail.com 


\section{INTRODUÇÃO}

A toxoplasmose é uma das zoonoses mais disseminadas do mundo, conhecida popularmente como a "doença do gato" e tem como agente etiológico o Toxoplasma gondii ( $T$. gondii). Esse protozoário possui dois tipos de hospedeiros: o intermediário e o definitivo. Como hospedeiros intermediários estão todos vertebrados homeotérmicos (aves e mamíferos) e como hospedeiros definitivos estão os felídeos ${ }^{1}$. Os felídeos assumem importância significativa na transmissão desse parasita, sendo os únicos hospedeiros que eliminam oocistos em suas fezes, contaminando o ambiente ${ }^{2}$, além disso, o gato doméstico possui um papel importante no ciclo da doença devido a sua presença no meio social.

A toxoplasmose pode ser transmitida de diversas formas, dentre elas está a ingestão de oocistos infectantes presentes na água e alimentos ou através mãos contendo fezes contaminadas de felinos, ingestão de cistos em carne crua ou mal cozida, transmissão congênita ou inoculação acidental de taquizoítos ${ }^{3}$. Para prevenir a transmissão congênita mulheres grávidas devem lavar as mãos ao manipular carne crua, lavar bem frutas e legumes, evitar o consumo de carne crua ou mal cozida, água não filtrada, assim como de alimentos expostos a insetos. Devem evitar também o contato com o solo, e ao lidar com materiais potencialmente contaminados com fezes de gatos, como a caixa de areia, é necessário o uso de luvas apropriadas ${ }^{4}$.

A infecção em hospedeiros definitivos ou intermediários é recorrente, no entanto, raramente apresentam sinais clínicos, e quando presentes, os sintomas dependem do estado imunológico do indivíduo, quantidade de microrganismos ingeridos, e se há presença de uma doença concomitante ${ }^{5}$. Embora a infecção seja geralmente assintomática nos indivíduos imunocompetentes, costuma apresentar quadros clínicos de alta gravidade em indivíduos imunocomprometidos, podendo até levar à morte ${ }^{4}$. $\mathrm{Na}$ toxoplasmose adquirida, os sintomas mais comuns em imunocompetentes levam a um quadro semelhante à Mononucleose infecciosa, com adenopatias, principalmente cervicais, frequentemente acompanhadas de febre baixa, desâmino e anorexia ${ }^{3}$.

A infecção materna primária com $T$. gondii adquirida durante a gestação, é de elevada importância pelo fato de poder resultar em infecção com graves sequelas para o feto ${ }^{6}$. Durante o primeiro trimestre da gestação, a infecção pode levar à morte fetal, no segundo trimestre, pode ocasionar a chamada Tétrade de Sabin, em que o feto apresenta retinocoroidite, calcificações cerebrais, retardo mental ou perturbações neurológicas e alterações do volume craniano com macro ou microcefalia, quando a infecção é adquirida no terceiro trimestre de gestação, a criança pode nascer normal e apresentar evidências da doença alguns dias, semanas ou meses após o parto7.

A transmissão congênita acontece através da passagem transplacentária do T. gondii, quando a gestante contrai a infecção, próximo ou durante a gestação, ou raramente, quando a doença se manifesta novamente de forma aguda, quando gestantes cronicamente infectadas tem imonucomprometimento significativo ${ }^{3,8}$. É importante verificar se a infecção ocorreu durante a gestação, pois, como descrito, é nessa situação que há risco de transmissão do T. gondii para o feto ${ }^{4}$.

A toxoplasmose congênita é considerada importante causa mundial de morbidade e mortalidade infantil ${ }^{6}$. Aproximadamente $15 \%$ das infecções fetais resultam em morte intrauterina. Dos fetos que nascem $80 \%$ desenvolvem lesões oculares e desordens cerebrais tardias ao longo da vida ${ }^{3}$. Assim, a prevenção da doença assume importância fundamental, uma vez que as sequelas são irreversíveis 9 .

A prevalência de soropositividade em gestantes varia conforme regiões geográficas, características climáticas, fatores culturais e hábitos alimentares ${ }^{10}$. Dependendo das condições de saneamento e socioeconômica, a taxa de prevalência de toxoplasmose pode alcançar até 90\% da população adulta ${ }^{11}$.

Diante disso, o objetivo deste estudo foi analisar a prevalência de toxoplasmose em gestantes que realizaram o pré-natal através do Sistema Único de Saúde, em Divinópolis-MG, durante os dois últimos anos, relacionando a prevalência da doença em gestantes com a distribuição regional no município de Divinópolis, e associa-la com a idade materna das gestantes portadoras do de T. gondii.

\section{MATERIAIS E MÉTODOS}

Foi feito um estudo transversal retrospectivo com os resultados dos testes sorológicos para diagnóstico de toxoplasmose realizado em gestantes atendidas pelo Sistema Único de Saúde (SUS) durante os anos de 2013 e 2014, no município de Divinópolis-MG, Brasil.

O município de Divinópolis está situado na região centro-oeste de Minas Gerais, com população de 230.848 habitantes, área de $709 \mathrm{~km}^{2}$, da qual $524 \mathrm{~km}^{2}$ é rural, que, devido à sua localização, desenvolvimento econômico e organização do seu sistema de saúde, é um centro de macrorregionais para 54 cidades vizinhas ${ }^{12,13}$.

O Programa de Controle da Toxoplasmose Congênita em Minas Gerais é coordenado pela Secretaria de Estado de Saúde (SES-MG) e faz parte do Programa Mães de Minas, voltado para a proteção e cuidado da gestante e da criança 
no primeiro ano de vida. Dessa forma, o Estado garante que todas as gestantes realizem gratuitamente os testes para o diagnóstico precoce da toxoplasmose, assim como o tratamento dos casos identificados ${ }^{14}$.

Os dados obtidos a partir desse programa, referentes ao município, são arquivados na Secretaria Municipal de Saúde. Dessa forma, para realização do presente estudo, foram solicitados, à Secretaria, os dados da população de gestantes assistidas pelo SUS no município de Divinópolis-MG, durante os anos de 2013 e 2014. O método para diagnóstico utilizado durante os anos estudados foi o ELISA, na análise de dados foram considerados resultados negativos os testes que acusaram IgM e IgG não reagentes, resultados positivos em fase crônica e aguda, este os testes que acusaram IgM e IgC reagentes e aquele os que apresentaram IgM não reagente e $\operatorname{lgG}$ reagente.

Todos os testes realizados para toxoplasmose em gestantes no período de janeiro de 2013 a dezembro de 2014 foram incluídos, e a partir dos resultados positivos, foram distinguidas as pacientes com a fase aguda e crônica da doença toxoplasmose em cada ano.

Os endereços das gestantes foram utilizados na análise comparativa dos casos de toxoplasmose entre as 11 regiões da cidade, fazendo assim, uma relação entre distribuição regional das grávidas soropositivas para toxoplasmose. O mapa com as regiões utilizadas nesse estudo está de acordo com o Plano Diretor Participativo de Divinópolis ${ }^{15}$ (2013) e foi adaptado com os índices encontrados no presente estudo. As idades das gestantes foram organizadas em seis categorias como se segue: 13-14 anos; 15-19 anos; 20-24 anos; 25-29 anos; 30-34 anos; e $\geq 35$ anos, para realizar a relação entre idade e prevalência da toxoplasmose na população estudada.

O presente estudo foi aprovado pelo Comitê de Ética da Universidade do Estado de Minas Gerais, parecer noㅡ 1.050.416.

Os dados foram descritos pelo número de ocorrências e porcentual, a faixa etária e as diferenças entre as regiões analisadas pelo teste de qui-quadrado, através do software SPSS 15.0 for Windows. Foram considerados como diferença significativa valores nos quais $p<0,05$. A proporção de positividade (PP) por região e por categoria de idade se dá pela relação entre o número de exames positivos (EP) por região e o total de exames realizados (TE) na mesma região, através da aplicação da fórmula $\mathrm{PP}=(\mathrm{EP} / \mathrm{TE})^{*} 100$.

\section{RESULTADOS}

Em Divinópolis, durante o ano de 2013 foram realizados 691 exames para detecção de toxoplasmose em gestantes, nos quais foi encontrada a prevalência de $45 \%, 54 \%$ das gestantes encontravam-se suscetíveis, acusando sorologia negativa e $1 \%$ do total de exames apresentou resultado inconclusivo para infecção aguda e não foram repetidos para confirmação. Do total de exames positivos, em 99,7\% $(n=310)$ dos casos as gestantes apresentaram fase crônica e $0,3 \%(n=1)$ acusou sorologia conclusiva para infecção aguda.

Em 2014, foram realizados 1343 exames, sendo observado $38 \%$ dos casos com sorologia positiva, $1 \%$ dos exames foram inconclusivos para infecção aguda e não foram repetidos para confirmação, portanto, $61 \%$ dos exames acusaram sorologia negativa. Do total de casos positivos 99,2\% $(n=509)$ das gestantes estavam cronicamente infectadas e $0,8 \%(n=4)$ apresentaram resultado conclusivo para infecção aguda. Devido ao pequeno número de gestantes em fase aguda durante os dois anos não foi possível realizar análise estatística.

Quanto à distribuição regional da prevalência de T. gondii nas gestantes no município de Divinópolis-MG em 2013, os locais que apresentaram maior frequência foram as regiões Oeste, Sudoeste distante e Noroeste rural com 58,6, 58,3 e $57,1 \%$, respectivamente (Tabela 1 e Figura 1). Não foi encontrada diferença significativa entre as regiões $(p>0,05)$.

Durante o ano de 2014, o resultado foi semelhante ao ano de 2013, as regiões que apresentaram maior frequência foram Noroeste distante, Sudoeste distante e Oeste com $51,1,45,3$ e $45 \%$, respectivamente (Figura 1). Do total de exames, três não tinham disponíveis os dados sobre endereço. Não foi encontrada diferença significativa entre as regiões $(p>0,05)$.

Ao associar a frequência de $T$. gondii com a faixa etária das gestantes, na faixa de 13-14 anos foi encontrada soropositividade de $100 \%$, no entanto, a amostra foi de apenas uma gestante. Com exceção da 13-14, as categorias que apresentaram maiores prevalências foram de gestantes com idade $\geq 35,30-34$ e 15-19 anos, sendo encontrados 58, 55 e 40\%, respectivamente (Tabela 1). Três exames não continham informações sobre idade, destes um resultado acusou sorologia positiva. Esses índices se dão em relação aos casos positivos das categorias de idade sobre o total de exames realizados por categoria correspondente, sendo encontrada diferença significativa durante o ano de 2013 $(p<0,05)$.

Em 2014, semelhante a 2013, a categoria de 13-14, apresentou a amostragem reduzida, constatando a prevalência de 42,9\%. Em seguida as categorias com maiores prevalências foram $\geq 35,30-34$ e 20-24 anos, sendo encontrados 42,8, 42,3 e 37,2\%, respectivamente. Os valores absolutos e proporcionais estão disponíveis na Tabela 1. Não houve diferença significativa $(p>0.05)$. 
Tabela 1. Prevalência de Toxoplasma gondii em gestantes atendidas pelo SUS, durante os anos de 2013 e 2014 em Divinópolis (MG, Brasil), em diferentes categorias de idade.

\begin{tabular}{|c|c|c|c|c|c|c|}
\hline \multirow[t]{2}{*}{ Categoria de idade } & \multicolumn{2}{|c|}{ Total de exames realizados* } & \multicolumn{2}{|c|}{ Exames positivos* } & \multicolumn{2}{|c|}{$\begin{array}{l}\text { Proporção de positividade } \\
\text { por categoria de idade (\%) }\end{array}$} \\
\hline & 2013 & 2014 & 2013 & 2014 & 2013 & 2014 \\
\hline $13-14$ & 1 & 7 & 1 & 3 & 100 & 42,9 \\
\hline $15-19$ & 100 & 205 & 40 & 76 & 40 & 37,1 \\
\hline $20-24$ & 179 & 352 & 67 & 131 & 37,4 & 37,2 \\
\hline $25-29$ & 156 & 318 & 60 & 107 & 38,5 & 33,6 \\
\hline $30-34$ & 140 & 246 & 77 & 104 & 55 & 42,3 \\
\hline$\geq 35$ & 112 & 215 & 65 & 92 & 58 & 42,8 \\
\hline Sem idade & 3 & 0 & 1 & 0 & 33,3 & 0 \\
\hline Total & 691 & 1343 & 311 & 513 & & \\
\hline
\end{tabular}

* Valores absolutos.

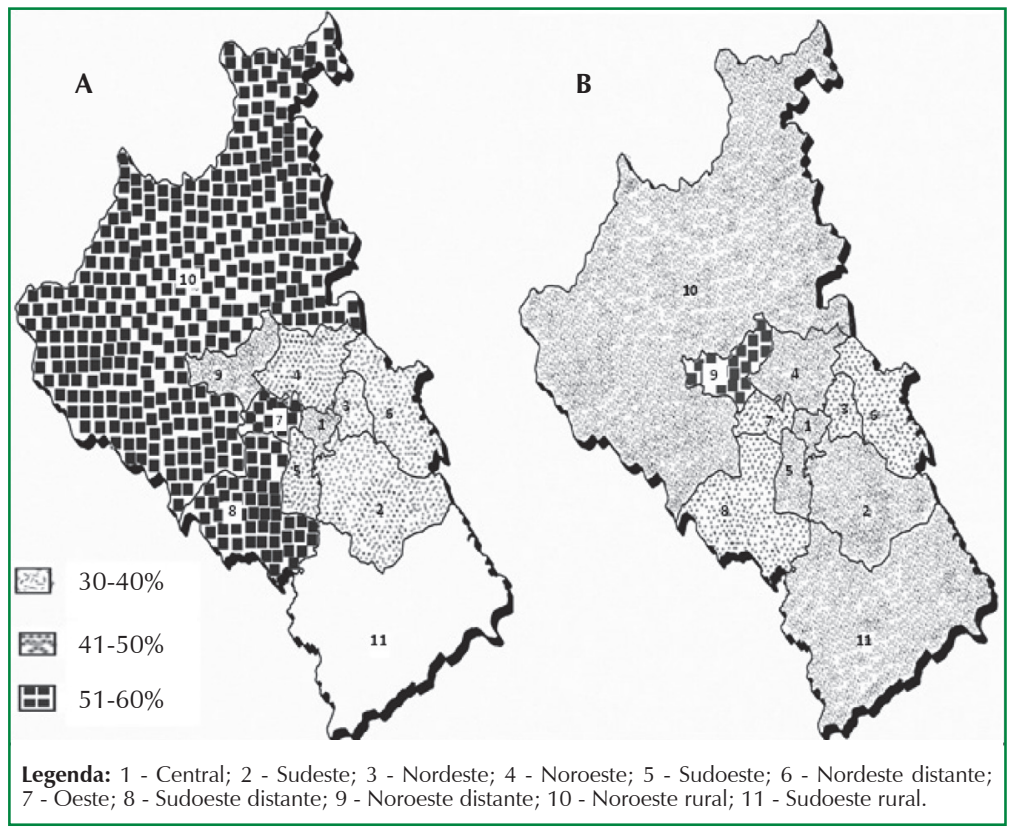

Figura 1. Mapa da distribuição da soropositividade de Toxoplasma gondii nas regiões de Divinópolis (MG, Brasil), durante os anos de 2013 (A) e 2014 (B), respectivamente.
A faixa etária das gestantes atendidas entre os dois anos variou de 14 a 61 anos, as que apresentaram maior frequência estavam entre $15 \mathrm{a} \geq 35$ anos. Mulheres em idade fértil estão na faixa etária de 10 a 49 anos, e de 15 a 49 anos internacionalmente ${ }^{16}$. A idade da gestante com 61 anos, encontrada no presente estudo pode ser justificada por erro de digitação durante a coleta de informações da triagem. As gestantes com sorologia conclusiva para fase aguda da doença apresentaram maior frequência na faixa etária de 15 a 19 anos, frequência de 100\%, num total de cinco gestantes.

\section{DISCUSSÃO}

Foram encontradas prevalências de 45 e 38\% para toxoplasmose, durante 2013 e 2014, respectivamente. Em outro estudo realizado anteriormente em Divinópolis foi registrado 49,5\% de gestantes infectadas na fase crônica, durante o período estudado nos anos de 2007 e $2008^{13}$. As prevalências de $T$. gondii observadas na cidade foram semelhantes às de outras localidades do Brasil, como no Pontal do Triângulo Mineiro que encontrou soroprevalência de $36 \%{ }^{17}$, este, por sua vez, foi semelhante à Caxias do SulRS nos quais os índices de toxoplasmose atingiram 36,8\% nas mulheres estudadas, destas $31 \%$ eram gestantes ${ }^{18}$. Por outro lado, os resultados encontrados no presente estudo estão menores ao do Estado do Mato Groso do Sul, onde a prevalência foi de $50,20 \%{ }^{3}$ e o munícipio de Porto Alegre-RS, que encontrou prevalência de 59,8\% nas gestantes estudadas $^{10}$. No entanto, algumas localidades possuem índices ainda mais altos de infecção, como no estudo realizado na população da zona rural de Jaguapitã-PR, 
na qual a prevalência foi de $66 \%{ }^{19}$; gestantes triadas em Goiânia-GO, prevalência de $67,7 \%{ }^{20}$; Porto Velho-RO, $73,4 \%^{21}$; gestantes atendidas pela Rede Municipal de Saúde de Vitória-ES, com taxa de $73,5 \%{ }^{22}$; e em gestantes atendidas no sistema público de saúde em Caxias-MA, onde a prevalência foi de $77,9 \%{ }^{23}$.

A amplitude nas prevalências entre estudos distintos pode estar relacionada com a população selecionada, com os diferentes métodos diagnósticos utilizados ou ainda a diferentes fatores de risco envolvidos na transmissão da doença, como condições sanitárias e índices socioeconômicos, tipo de alimentação, tratamento adequado da água e exposição ambiental ${ }^{4,9,11}$. Outros fatores que corroboram as diferentes taxas de prevalência da toxoplasmose são a grande extensão territorial, diversidade sociocultural e nível de instrução ${ }^{24}$.

Foram encontradas 0,3 e $0,8 \%$ de gestantes em fase ativa da doença em 2013 e 2014, respectivamente, em Divinópolis-MG, resultado próximo de Caxias-MA, onde foi encontrada prevalência de $0,9 \%$ de infecção ativa nas gestantes estudadas ${ }^{23}$. Espera-se que as infecções agudas ocorram mais frequentemente em pacientes mais jovens, devendo-se dar cuidado excepcional a estas gestantes, considerando que as mesmas têm risco maior de adquirir toxoplasmose, pois quanto menor a idade, menor o tempo de exposição aos fatores de risco ${ }^{25}$. Os resultados encontrados corroboram essa afirmativa, já que todas as gestantes que apresentaram resultado conclusivo para infecção aguda durante anos estudados apresentaram maior frequência na faixa etária de 15 a 19 anos.

Entre os dois anos analisados houve diferença significativa, atribuída ao aumento considerável de gestantes atendidas durante o último ano pelo SUS, em DivinópolisMG. É importante ressaltar que 54 e $61 \%$ das gestantes encontravam-se suscetíveis para infecção durante 2013 e 2014, respectivamente, demonstrando a necessidade da implantação de medidas de prevenção primária devido à possibilidade de ocorrer a soroconversão e infecção aguda durante o período gestacional ${ }^{25}$.

Não foi encontrada associação significativa entre a distribuição regional e soroprevalência de $T$. gondii nas gestantes. No entanto, foram observados valores maiores da frequência do parasita em gestantes de algumas regiões da cidade em comparação as outras. Esses valores podem estar relacionados aos diferentes comportamentos da população e às condições socioeconômicas e de saneamento desses locais, fazendo com que as pessoas fiquem mais expostas aos fatores de riscos ${ }^{25}$. Outro elemento encontrado anteriormente na cidade que pode ser associado à variação de registro do parasita entre as regiões foi a menor procura ao serviço de saúde pública pelas gestantes em algumas regiões ${ }^{13}$. A redução na utilização dos serviços de saúde pública, em grande parte, resulta em falta de informação sobre a doença e seu respectivo modo profilático, ocasionando índices maiores dos parasitas em determinados lugares.

De maneira geral, foi possível perceber que os maiores valores de prevalência estão entre $15 \mathrm{a} \geq 35$ anos. No ano de 2013 foi encontrada associação entre a soroprevalência e o acréscimo da idade, semelhante ao encontrado em Goiânia$\mathrm{GO}$, na qual a faixa predominante foi de 20 a 30 anos, aumentando com a idade ${ }^{20}$. Em Porto Velho-RO, verificaram maior prevalência nos pacientes que se encontravam entre as faixas etárias de 16 a 35 anos $^{21}$. Enquanto em Caxias do Sul-RS, a maior frequência foi encontrada entre a faixa etária de 37 a 49 anos $^{18}$. Estudos que corroboram o encontrado, demonstram associação entre a soroprevalência e o incremento da idade, fato que pode ser explicado pelo maior tempo de exposição aos fatores de risco 3,10,25.

Estudo realizado anteriormente a esse por Fonseca et al. ${ }^{13}$ em 2012, na mesma cidade, demonstrou que 93\% das gestantes entrevistadas possuíam pouco ou nenhum conhecimento sobre a toxoplasmose, concluindo que a falta de informações sobre a doença foi o fator mais relevante para soroprevalência e isso influencia diretamente a transmissão e disseminação da doença. Dessa forma, as campanhas de sensibilização devem ser realizadas para evitar o aumento de toxoplasmose bem como outras parasitoses ${ }^{13}$. Destaca-se ainda, que os programas de educação podem não só proteger a população como reduzir despesas financeiras sobre os tratamentos de pacientes acometidos por essa doença, fazendo da educação parte das políticas de saúde ${ }^{13}$.

Foi possível concluir que os valores encontrados em Divinópolis estão dentro dos valores verificados em outros estudos no Brasil, demonstrando que durante os dois anos um alto nível de gestantes apresentou contato prévio com o parasita, e baixo nível de infecção recente. No entanto, as gestantes soronegativas apresentavam risco para infecção e possível transmissão fetal, demonstrando assim, que as medidas preventivas adotadas no serviço público de saúde devem ser mantidas.

A partir da distribuição da toxoplasmose pelas regiões de Divinópolis é possível inferir em quais regiões existe a necessidade de intensificar as estratégias, como palestras educativas em saúde e acompanhamento às gestantes. Destaca-se, que as estratégias preventivas devem ser realizadas durante todo o acompanhamento gestacional, ressaltando os fatores risco e medidas de prevenção, para todas gestantes e com atenção especial às gestantes suscetíveis, assim como no início da vida reprodutiva da mulher, já que a possibilidade de adquirir a forma aguda é maior nesse momento. E dessa forma, reduzir a possibilidade de soroconversão e transmissão fetal durante a gestação. 


\section{AGRADECIMENTOS}

À Secretaria Municipal de Saúde, por fornecer os dados essenciais para a conclusão deste estudo. À Universidade do Estado de Minas Gerais (UEMG) pela bolsa do Programa Institucional de Apoio à pesquisa (PAPq) e pela estrutura física e apoio fornecido durante o desenvolvimento da pesquisa.

\section{REFERÊNCIAS}

1. Kawasoe U. Toxoplasma gondii. In: Neves DP, Melo AL, Linardi PM, Vitor RWA. Parasitologia Humana. 11a ed. São Paulo: Atheneu; 2005. p. 163-72.

2. Climeni BSO, Monteiro MV, Neves, MF. Toxoplasmose. Rev Cient Eletr Med Vet [Internet]. 2009 [citado em 2017];7(12). Disponível em: http://faef.revista.inf.br/imagens_arquivos/arquivos_destaque/ akl1SBAAx865DbJ_2013-6-21-16-10-17.pdf

3. Oliveira AC, Martins DOO, Silva EC, Botelho JAO. Prevalência de toxoplasmose em gestantes triadas pelo programa estadual de proteção a gestantes de Mato Grosso Do Sul, no período de 2011. In: IV Seminário de Pesquisa e TCC da FUG, 2012. Trindade-GO: Faculdade União de Goyazes; 2012. p. 81-102.

4. Amendoeira MRR, Coura LFC. Uma breve revisão sobre toxoplasmose na gestação. Sci. Med. 2010;20(1):113-19.

5. Negri D, Cirilo MB, Salvarani RS, Neves MF. Toxoplasmose em cães e gatos. Rev Cient Eletr Med Vet [Internet]. 2008 [citado em 2017];6(11). Disponível em: http://faef.revista.inf.br/imagens_arquivos/arquivos_ destaque/PInrs8cwKOYc3nV_2013-6-13-15-16-0.pdf

6. Silva LR, Okazaki ELFJ. Enfermagem e a prevenção da toxoplasmose durante a gestação. Rev Enferm UNISA. 2012;13(1):43-7.

7. Souza CO, Tashima NT, Silva MA, Tumitan ARP. Estudo transversal de toxoplasmose em alunas de um curso superior da região de Presidente Prudente, Estado de São Paulo. Rev Soc Bras Med Trop. 2010;43(1):59-61. https://doi.org/10.1590/S003786822010000100013

8. Silva MG, Castro AM. Fatores epidemiológicos e triagem neonatal associados à toxoplasmose gestacional e congênita em Gurupi, Tocantins [tese]. Goiânia: Universidade Federal de Goiás; 2014.

9. Varella IRS, Wagner MB. Prevalência de toxoplasmose aguda em gestantes, incidência de toxoplasmose congênita e desempenho de testes diagnósticos em toxoplasmose congênita [tese]. Porto Alegre: Universidade Federal do Rio Grande do Sul; 2007.

10. Varella IRS, Wagner MB, Darela AC, Nunes LM, Muller RW. Prevalência de soropositividade para toxoplasmose em gestantes. J Pediatr (Rio J). 2003;79(1):69-74. https://doi.org/10.1590/s002175572003000100012

11. Vaz RS, Rauli P, Mello RG, Cardoso MA. Toxoplasmose congênita: uma doença negligenciada? Política pública de saúde atual brasileira. FACTS. 2011;(3):1-8.

12. IBGE - Instituto Brasileiro de Geografia e Estatística [Internet]. [citado em 2015 Out. 15]. Disponível em: http://www.cidades. ibge.gov.br/xtras/perfil.php?lang $=\&$ codmun $=312230 \&$ search $=\mathrm{mi}$ nas-gerais | divinopolis
13. Fonseca AL, Silva RA, Fux B, Madureira AP, Sousa FF, Margonari C. Epidemiologic aspects of toxoplasmosis and evaluation of its seroprevalence in pregnant women. Rev Soc Bras Med Trop. 2012;45(3):357-64. https://doi.org/10.1590/S003786822012000300015

14. Núcleo de Ações e Pesquisa em Apoio Diagnóstico. Programa de Controle da Toxoplasmose Congênita em Minas Gerais [Internet]. 2015 [citado em 2015].

15. Plano Diretor Participativo de Divinópolis. Mapa das regiões de Divinópolis [Internet]. 2013 [citado em 2015 jun. 5]. Disponível em: https://planodiretordedivinopolis.wordpress.com/category/ divinopolis/

16. Ferreira DL, Pires VATN. Perfil de morbidade e mortalidade de mulheres em idade fértil na área de abrangência da microrregião de saúde de Ipatinga. Rev Enferm Int. 2013;6(1):1119- 32.

17. Maia LP, Hérnandez CG, Oliveira KR, Nomeline QSS, Aidar FLM, Ferreira GLS. Soroprevalência de toxoplasmose na região do pontal do triângulo mineiro, Minas gerais, Brasil. Rev Patol Trop. 2012;41(4):457-64. https://doi.org/10.5216/rpt.v41i4.21707

18. Detanico L, Basso RMC. Toxoplasmose: perfil sorológico de mulheres em idade fértil e gestantes. Rev Bras Anal Clín. 2006; 38(1):15-8

19. Garcia JL, Navarro IT, Ogawa L, Oliveira RC, Kobilka E. Soroprevalência, epidemiologia e avaliação ocular da toxoplasmose humana na zona rural de Jaguapitã (Paraná), Brasil. Rev Panam Salud Públ. 1999;6(3):157-63. https://doi.org/10.1590/S102049891999000800002

20. Sartori AL, Minamisava R, Avelino MM, Martins CA. Triagem prénatal para toxoplasmose e fatores associados à soropositividade de gestantes em Goiânia, Goiás. Rev Bras Ginecol Obstet. 2011;33(2): 93-8. https://doi.org/10.1590/S0100-72032011000200007

21. Foschiera AIC, Cartonilho G, Teles CBG. Prevalência da toxoplasmose em pacientes atendidos no laboratório central de saúde pública de Porto Velho-RO. RESC. 2009;2(1):92-103.

22. Areal KR; Miranda AE. Soroprevalência de toxoplasmose em gestantes atendidas na rede básica de saúde de Vitória, ES. Rev Newslab. 2008;87:122-29.

23. Câmara JT, Silva MG, Castro AM. Prevalência de toxoplasmose em gestantes atendidas em dois centros de referência em uma cidade do Nordeste, Brasil. Rev Bras Ginecol Obstet. 2015;37(2):64-70. https://doi.org/10.1590/SO100-720320150005115

24. Bittencourt LHFB, Mori FMRL, Breganó RM, Zabott MV, Freire RL, Pinto SB, Navarro IT. Soroepidemiologia da toxoplasmose em gestantes a partir da implantação do Programa de Vigilância da Toxoplasmose Adquirida e Congênita em municípios da região oeste do Paraná. Rev Bras Ginecol Obstet. 2012;34(2): 63-8.

25. Inagaki AD, Cardoso NP, Lopes RJ, Alves JA, Mesquita JR, Araújo KC, Katagiri S. Análise espacial da prevalência de toxoplasmose em gestantes de Aracaju, Sergipe, Brasil. Rev Bras Ginecol Obstet. 2014;36(12):535-40. https://doi.org/10.1590/So100720320140005086 